\title{
ALMOST EVERYWHERE CONVERGENCE OF VILENKIN-FOURIER SERIES OF $H^{1}$ FUNCTIONS
}

\author{
WO-SANG YOUNG
}

(Communicated by J. Marshall Ash)

\begin{abstract}
In [5] Ladhawala and Pankratz proved that if $f$ is in dyadic $H^{1}$, then any lacunary sequence of partial sums of the Walsh-Fourier series of $f$ converges a.e. We generalize their theorem to Vilenkin-Fourier series. In obtaining this result, we prove a vector-valued inequality for the partial sums of Vilenkin-Fourier series.
\end{abstract}

\section{INTRODUCTION}

Let $G=\prod_{i=0}^{\infty} Z_{p_{i}}$ be the countable direct product of cyclic groups of order $p_{i}$, where $\left\{p_{i}\right\}_{i>0}$ is a sequence of integers with $p_{i} \geq 2$, and $\mu$ be the Haar measure on $G$ normalized by $\mu(G)=1$. $G$ can be identified with the unit interval $(0,1)$ in the following manner. Set $m_{0}=1, m_{k}=\prod_{i=0}^{k-1} p_{i}$, $k=1,2, \ldots$. We associate with each $\left\{x_{i}\right\} \in G, 0 \leq x_{i}<p_{i}$, the point $\sum_{i=0}^{\infty} x_{i} m_{i+1}^{-1} \in(0,1)$. If we disregard the countable set of $p_{i}$-rationals, this mapping is one-one, onto, and measure-preserving.

For $x=\left\{x_{i}\right\} \in G$, let $\phi_{k}(x)=\exp \left(2 \pi i x_{k} / p_{k}\right), k=0,1, \ldots$ We consider all finite products $\left\{\chi_{n}\right\}$ of $\left\{\phi_{k}\right\}$, enumerated according to a scheme of Paley. We express each nonnegative integer $n$ as a finite sum $n=\sum_{k=0}^{\infty} \alpha_{k} m_{k}$, with $0 \leq \alpha_{k}<p_{k}$, and define $\chi_{n}=\prod_{k=0}^{\infty} \phi_{k}^{\alpha_{k}}$. The functions $\left\{\chi_{n}\right\}$ are the characters of $G$, and they form a complete orthonormal system on $G$. For the case $p_{i}=2$, $i=0,1, \ldots, G$ is the dyadic group, $\left\{\phi_{k}\right\}$ are the Rademacher functions, and $\left\{\chi_{n}\right\}$ are the Walsh functions. In general, the system $\left(G,\left\{\chi_{n}\right\}\right)$ is a realization of the Vilenkin systems studied in [7].

We consider the Fourier series with respect to $\left\{\chi_{n}\right\}$. For $f \in L^{1}(G)$, let

$$
S_{n} f(x)=\int_{G} f(t) \sum_{j=0}^{n-1} \chi_{j}(x-t) d \mu(t), \quad n=1,2, \ldots,
$$

Received by the editors March 31, 1989.

1980 Mathematics Subject Classification (1985 Revision). Primary 42C10; Secondary 42A20, 42B 30 . 
be the $n$th partial sum of the Vilenkin-Fourier series of $f$. We define $H^{1}$ in terms of the $m_{k}$ th partial sums $S_{m_{k}} f$, which are special for the VilenkinFourier series. Let $\left\{G_{k}\right\}$ be a sequence of subgroups of $G$ defined by

$$
G_{0}=G, \quad G_{k}=\prod_{i=0}^{k-1}\{0\} \times \prod_{i=k}^{\infty} Z_{p_{i}}, \quad k=1,2, \ldots,
$$

and $\mathscr{F}_{k}$ be the $\sigma$-algebra generated by the cosets of $G_{k}$. On the interval $(0,1)$, atoms of $\mathscr{F}_{k}$ are intervals of the form $\left(j m_{k}^{-1},(j+1) m_{k}^{-1}\right), j=0,1, \ldots, m_{k}-$ 1. We note that $\left\{\mathscr{F}_{k}\right\}$ is an increasing sequence of $\sigma$-algebras. Since $S_{m_{k}} f$ is the average of $f$ over the cosets of $G_{k}$ (see, e.g. [8, p. 312]), $\left\{S_{m_{k}} f, \mathscr{F}_{k}\right\}$ is a martingale.

For $f \in L^{1}(G)$, let $f^{*}=\sup _{k \geq 0}\left|S_{m_{k}} f\right|$ and

$$
S(f)=\left[\sum_{k=-1}^{\infty}\left(S_{m_{k+1}} f-S_{m_{k}} f\right)^{2}\right]^{1 / 2}, \quad \text { where } S_{m_{-1}} f=0
$$

Applying Davis' result for martingales [1], we know that there exist positive constants $c$ and $C$ (independent of the orders $\left\{p_{i}\right\}$ ) such that

$$
c\|S(f)\|_{1} \leq\left\|f^{*}\right\|_{1} \leq C\|S(f)\|_{1} .
$$

We say that $f \in H^{1}(G)$ if $S(f) \in L^{1}(G)$, or, equivalently, $f^{*} \in L^{1}(G)$, and we write

$$
\|f\|_{H^{1}}=\|S(f)\|_{1} \text {. }
$$

Our definition of $H^{1}(G)$ is a special case of the definition of $H^{1}$ for martingales given by Garsia [3]. For other definitions of $H^{1}(G)$, see [2] and [6].

We have the following theorem concerning the a.e. convergence of VilenkinFourier series of functions in $H^{1}(G)$.

Theorem 1. Let $f \in H^{1}(G)$ and let $\left\{n_{k}\right\}_{k \geq 0}$ be a sequence of positive integers such that $m_{k} \leq n_{k}<m_{k+1}, k=0,1, \ldots$ Then, as $k \rightarrow \infty, S_{n_{k}} f(x) \rightarrow f(x)$ for a.e. $x \in G$.

For the case where $G$ is the dyadic group, the theorem is proved by Ladhawala and Pankratz [5]. If $f \in L^{p}(G), 1<p<\infty$, then $f^{*} \in L^{p}(G)$ by Doob's inequality. Hence $L^{p}(G) \subset H^{1}(G)$. Even for the case where $f \in L^{p}(G), 1<p<\infty$, our result is new if the orders of the cyclic groups are unbounded, i.e. $\sup _{i} p_{i}=\infty$. For the bounded case, $\sup _{i} p_{i}<\infty$, Gosselin [4] showed that if $f \in L^{p}(G), 1<p<\infty$, the full sequence of partial sums $\left\{S_{n} f\right\}$ converges a.e. to $f$.

To prove Theorem 1, we shall show that, for any sequence $\left\{n_{k}\right\}$ with $m_{k} \leq$ $n_{k}<m_{k+1}, k=0,1, \ldots$, we have

$$
\mu\left\{x \in G: \sup _{k \geq 0}\left|S_{n_{k}} f(x)\right|>y\right\} \leq C y^{-1}\|f\|_{H^{1}},
$$


where $y>0, f \in H^{1}(G)$, and $C$ is an absolute constant independent of the orders $\left\{p_{i}\right\}$. Since $S_{m_{k}} f$ converges to $f$ in the $H^{1}$ norm, Theorem 1 will then follow by the usual density argument.

We shall obtain (1.2) as a consequence of a vector-valued inequality concerning the partial sums of Vilenkin-Fourier series.

Theorem 2. There exist constants $C$ and $C_{p}$ such that, for any sequence $\left\{f_{l}\right\}$ of functions in $L^{1}(G)$ and any sequence of positive integers $\left\{n_{l}\right\}$,

$$
\begin{aligned}
& \mu\left\{x \in G:\left(\sum_{l=0}^{\infty}\left|S_{n_{l}} f_{l}(x)\right|^{2}\right)^{1 / 2}>y\right\} \leq C y^{-1}\left\|\left(\sum_{l=0}^{\infty}\left|f_{l}\right|^{2}\right)^{1 / 2}\right\|_{1}, \quad y>0, \\
& \text { (1.4) }\left\|\left(\sum_{l=0}^{\infty}\left|S_{n_{l}} f_{l}\right|^{2}\right)^{1 / 2}\right\|_{p} \leq C_{p}\left\|\left(\sum_{l=0}^{\infty}\left|f_{l}\right|^{2}\right)^{1 / 2}\right\|_{p}, \quad 1<p<\infty .
\end{aligned}
$$

The constants $C$ and $C_{p}$ are independent of the orders $\left\{p_{i}\right\}$ of the cyclic groups.

In what follows, $C$ will denote an absolute constant, which may vary from line to line.

\section{A DECOMPOSITION LEMMA}

To prove Theorem 2, we use a Calderón-Zygmund type decomposition lemma. This lemma is a modified version of the one given in [8; Lemma 2, p. 314]. We shall describe it on the interval $(0,1)$.

Lemma 3. Let $y>0$ and $\left\{f_{l}\right\}_{l \geq 0}$ be a sequence of functions on $G$ such that $\left\|\left(\sum_{l}\left|f_{l}\right|^{2}\right)^{1 / 2}\right\|_{1} \leq y$. Let $\left\{\alpha_{l k}\right\}_{l, k \geq 0}$ be a double sequence of integers with $0 \leq$ $\alpha_{l k}<p_{k}$. Then there are sequences of functions $\left\{g_{l}\right\}_{l \geq 0},\left\{b_{l}\right\}_{l \geq 0}$ on $G$ and a collection $\mathscr{C}=\left\{\omega_{j}\right\}$ of disjoint intervals such that

$$
\begin{gathered}
f_{l}=g_{l}+b_{l}, \quad l=0,1, \ldots \\
\left(\sum_{l}\left|g_{l}\right|^{2}\right)^{1 / 2} \leq C y \quad \text { a.e. } \\
\left\|\left(\sum_{l}\left|g_{l}\right|^{2}\right)^{1 / 2}\right\|_{1} \leq C\left\|\left(\sum_{l}\left|f_{l}\right|^{2}\right)^{1 / 2}\right\|_{1} . \\
\mathscr{C}=\bigcup_{k=0}^{\infty} \mathscr{C}_{k},
\end{gathered}
$$

where each $\omega_{j} \in \mathscr{C}_{k}$ is measurable with respect to $\mathscr{F}_{k+1}$, and is a proper subset of a coset of $G_{k}$.

$$
b_{l}(x)=0 \quad \text { if } \quad x \notin \Omega \equiv \bigcup_{j} \omega_{j}, \quad l=0,1, \ldots .
$$


For each $l=0,1, \ldots$,

$$
\int_{\omega_{j}} b_{l} d \mu=0 \text { for every } \omega_{j} \in \mathscr{C} \text {, and }
$$$$
\int_{\omega_{j}} b_{l} \phi_{k}^{\alpha_{l k}} d \mu=0 \quad \text { for every } \omega_{j} \in \mathscr{C}_{k}, k=0,1, \ldots
$$

$$
\begin{gathered}
\int_{\omega_{j}}\left(\sum_{l}\left|b_{l}\right|^{2}\right)^{1 / 2} d \mu \leq C \int_{\omega_{j}}\left(\sum_{l}\left|f_{l}\right|^{2}\right)^{1 / 2} d \mu \text { for every } \omega_{j} \in \mathscr{C} . \\
\sum_{j} \mu\left(\omega_{j}\right) \leq y^{-1}\left\|\left(\sum_{l}\left|f_{l}\right|^{2}\right)^{1 / 2}\right\|_{1}
\end{gathered}
$$

Proof. We apply the decomposition in [8] (see proof of Lemma 2, pp. 314$315)$ to the function $\left(\sum_{l}\left|f_{l}\right|^{2}\right)^{1 / 2}$ to obtain a collection $\mathscr{C}=\left\{\omega_{j}\right\}$ of disjoint intervals with the properties that

$$
\begin{gathered}
y<\frac{1}{\mu\left(\omega_{j}\right)} \int_{\omega_{j}}\left(\sum_{l}\left|f_{l}\right|^{2}\right)^{1 / 2} d \mu \leq 3 y, \quad \omega_{j} \in \mathscr{C}, \\
\left(\sum_{l}\left|f_{l}(x)\right|^{2}\right)^{1 / 2} \leq y, \quad \text { for a.e. } x \notin \Omega,
\end{gathered}
$$

and that $\mathscr{C}=\bigcup_{k=0}^{\infty} \mathscr{C}_{k}$, where $\left\{\mathscr{C}_{k}\right\}$ satisfies (2.4). The first inequality of (2.9) then implies (2.8).

Next, we decompose $f_{l}=g_{l}+b_{l}, l=0,1, \ldots$, with

$$
g_{l}(x)= \begin{cases}f_{l}(x) & \text { if } x \notin \Omega, \\ a_{l k j}+b_{l k j} \phi_{k}^{-\alpha_{l k}}(x) & \text { if } x \in \omega_{j} \in \mathscr{C}_{k},\end{cases}
$$

where $a_{l k j}, b_{l k j}$ are constants chosen in such a way that

$$
\int_{\omega_{j}} f_{l} d \mu=\int_{\omega_{j}}\left(a_{l k j}+b_{l k j} \phi_{k}^{-\alpha_{l k}}\right) d \mu
$$

and

$$
\int_{\omega_{j}} f_{l} \phi_{k}^{\alpha_{l k}} d \mu=\int_{\omega_{j}}\left(a_{l k j}+b_{l k j} \phi_{k}^{-\alpha_{l k}}\right) \phi_{k}^{\alpha_{l k}} d \mu .
$$

Then $b_{l}=g_{l}-f_{l}$ satisfies (2.5) and (2.6). Also, it follows from the proof of (25) in [8, pp. 315-317] that

$$
\left|g_{l}(x)\right| \leq \frac{C}{\mu\left(\omega_{j}\right)} \int_{\omega_{j}}\left|f_{l}\right| d \mu, \quad x \in \omega_{j}, \omega_{j} \in \mathscr{C} .
$$

Hence, by Minkowski's inequality for integrals, we have

$$
\left(\sum_{l}\left|g_{l}(x)\right|^{2}\right)^{1 / 2} \leq \frac{C}{\mu\left(\omega_{j}\right)} \int_{\omega_{j}}\left(\sum_{l}\left|f_{l}\right|^{2}\right)^{1 / 2} d \mu, \quad x \in \omega_{j}, \omega_{j} \in \mathscr{C} .
$$

This inequality, together with (2.9) and (2.10), implies (2.2), (2.3) and (2.7). 


\section{Proof of Theorem 2}

We shall prove (1.3). The case $p=2$ of (1.4) is a consequence of Plancherel's formula. For $1<p<2,(1.4)$ follows from (1.3) and the case $p=2$ by the vector-valued Marcinkiewicz interpolation theorem. The case $2<p<\infty$ then follows by a duality argument.

Instead of proving (1.3), we shall prove an equivalent inequality involving the modified partial sums $\left\{S_{n}^{*} f\right\}$. For $f \in L^{1}(G)$, let

$$
S_{n}^{*} f=\bar{\chi}_{n} S_{n}\left(f \chi_{n}\right), \quad n=1,2, \ldots
$$

(For the properties of $S_{n}^{*} f$, see [8, pp. 313-314].) (1.3) is equivalent to

$$
\mu\left\{x \in G:\left(\sum_{l=0}^{\infty}\left|S_{n_{l}}^{*} f_{l}(x)\right|^{2}\right)^{1 / 2}>y\right\} \leq C y^{-1}\left\|\left(\sum_{l=0}^{\infty}\left|f_{l}\right|^{2}\right)^{1 / 2}\right\|_{1},
$$

where $y>0,\left\{f_{l}\right\}$ is any sequence of functions in $L^{1}(G)$, and $\left\{n_{l}\right\}$ is any sequence of positive integers.

To prove (3.1), we can assume $\left\|\left(\sum_{l}\left|f_{l}\right|^{2}\right)^{1 / 2}\right\|_{1} \leq y$. For each $l=0,1, \ldots$, decompose $f_{l}$ as in Lemma 3. Since

$$
\begin{aligned}
& \mu\left\{\left(\sum_{l}\left|S_{n_{l}}^{*} f_{l}\right|^{2}\right)^{1 / 2}>y\right\} \\
& \quad \leq \mu\left\{\left(\sum_{l}\left|S_{n_{l}}^{*} g_{l}\right|^{2}\right)^{1 / 2}>y / 2\right\}+\mu\left\{\left(\sum_{l}\left|S_{n_{l}}^{*} b_{l}\right|^{2}\right)^{1 / 2}>y / 2\right\},
\end{aligned}
$$

(3.1) will be proved if we can show that each term on the right is bounded by $C y^{-1}\left\|\left(\sum_{l}\left|f_{l}\right|^{2}\right)^{1 / 2}\right\|_{1}$.

Using Plancherel's formula, we obtain

$$
\begin{aligned}
\mu\left\{\left(\sum_{l}\left|S_{n_{l}}^{*} g_{l}\right|^{2}\right)^{1 / 2}>y / 2\right\} & \leq C y^{-2}\left\|\left(\sum_{l}\left|S_{n_{l}}^{*} g_{l}\right|^{2}\right)^{1 / 2}\right\|_{2}^{2} \\
& \leq C y^{-2}\left\|\left(\sum_{l}\left|g_{l}\right|^{2}\right)^{1 / 2}\right\|_{2}^{2} \\
& \leq C y^{-1}\left\|\left(\sum_{l}\left|f_{l}\right|^{2}\right)^{1 / 2}\right\|_{1},
\end{aligned}
$$

by (2.2) and (2.3).

To estimate the second term in (3.2), we use the following notation. Let $\omega_{j} \in \mathscr{F}_{k+1}$, with $\omega_{j}$ contained in the coset $I$ of $G_{k}$. We consider $I$ as a circle and let $\omega_{j}^{*}$ denote the interval inside $I$ which contains $\omega_{j}$ at its center 
with $\mu\left(\omega_{j}^{*}\right)=3 \mu\left(\omega_{j}\right)$. Let $\Omega^{*}=\bigcup_{j} \omega_{j}^{*}$. Then, by $(2.8)$,

$$
\mu\left(\Omega^{*}\right) \leq 3 \sum_{j} \mu\left(\omega_{j}\right) \leq 3 y^{-1}\left\|\left(\sum_{l}\left|f_{l}\right|^{2}\right)^{1 / 2}\right\|_{1} .
$$

Therefore, it suffices to prove

$$
\mu\left\{x \notin \Omega^{*}:\left(\sum_{l}\left|S_{n_{l}}^{*} b_{l}(x)\right|^{2}\right)^{1 / 2}>y / 2\right\} \leq C y^{-1}\left\|\left(\sum_{l}\left|f_{l}\right|^{2}\right)^{1 / 2}\right\|_{1} .
$$

To do this, we express $S_{n_{l}}^{*} b_{l}$ in terms of the conjugate functions. Let $f \in$ $L^{1}(G)$. For $x \in\left\{x_{k}\right\} \in G, I=x+G_{k}$, define

$$
H_{k} f(x)=\frac{1}{2} \frac{1}{\mu(I)} \int_{I \cap\left\{x_{k} \neq t_{k}\right\}} f(t) \cot \left(\pi\left(x_{k}-t_{k}\right) / p_{k}\right) d \mu(t) .
$$

If $n_{l}=\sum_{k=0}^{\infty} \alpha_{l k} m_{k}, 0 \leq \alpha_{l k}<p_{k}$, it is shown in [8, pp. 313-314] that

$$
\begin{aligned}
S_{n_{l}}^{*} b_{l}(x)= & \sum_{k=0}^{\infty} \frac{\alpha_{l k}}{\mu(I)} \int_{I \cap\left\{x_{k}=t_{k}\right\}} b_{l}(t) d \mu(t) \\
& +\frac{1}{2} \sum_{k=0}^{\infty} \phi_{k}^{-\alpha_{l k}}(x) \frac{1}{\mu(I)} \int_{I \cap\left\{x_{k} \neq t_{k}\right\}} b_{l}(t) \phi_{k}^{\alpha_{l k}}(t) d \mu(t) \\
& -\frac{1}{2} \sum_{k=0}^{\infty} \frac{1}{\mu(I)} \int_{I \cap\left\{x_{k} \neq t_{k}\right\}} b_{l}(t) d \mu(t) \\
& +i \sum_{k=0}^{\infty} \phi_{k}^{-\alpha_{l k}}(x) H_{k}\left(b_{l} \phi_{k}^{\alpha_{l k}}\right)(x) \\
& -i \sum_{k=0}^{\infty} H_{k} b_{l}(x) .
\end{aligned}
$$

For $x \notin \Omega^{*},(2.5)$ and (2.6) imply that the first three terms on the right vanish. (See the explanation below (29) on p. 317 in [8].) Thus, it follows from Minkowski's inequality that, for $x \notin \Omega^{*}$,

$$
\begin{aligned}
{\left[\sum_{l}\left|S_{n_{l}}^{*} b_{l}(x)\right|^{2}\right]^{1 / 2} \leq } & \sum_{k=0}^{\infty}\left[\sum_{l}\left|H_{k}\left(b_{l} \phi_{k}^{\alpha_{l k}}\right)(x)\right|^{2}\right]^{1 / 2} \\
& +\sum_{k=0}^{\infty}\left[\sum_{l}\left|H_{k} b_{l}(x)\right|^{2}\right]^{1 / 2}
\end{aligned}
$$

(3.3) will be proved if we show

$$
\begin{gathered}
\mu\left\{x \notin \Omega^{*}: \sum_{k=0}^{\infty}\left[\sum_{l}\left|H_{k}\left(b_{l} \phi_{k}^{\alpha_{l / k}}\right)(x)\right|^{2}\right]^{1 / 2}>y / 4\right\} \\
\leq C y^{-1}\left\|\left(\sum_{l}\left|f_{l}\right|^{2}\right)^{1 / 2}\right\|_{1}
\end{gathered}
$$


and

$$
\begin{gathered}
\mu\left\{x \notin \Omega^{*}: \sum_{k=0}^{\infty}\left[\sum_{l}\left|H_{k} b_{l}(x)\right|^{2}\right]^{1 / 2}>y / 4\right\} \\
\leq C y^{-1}\left\|\left(\sum_{l}\left|f_{l}\right|^{2}\right)^{1 / 2}\right\|_{1} .
\end{gathered}
$$

We shall demonstrate (3.4). (3.5) can be proved similarly.

Suppose $x \notin \Omega^{*}$ and $I=x+G_{k}$. Again it follows from (2.5) and (2.6) that

$$
\begin{aligned}
H_{k}\left(b_{l} \phi_{k}^{\alpha_{l k}}\right)(x)= & \frac{1}{2} \frac{1}{\mu(I)} \sum_{\omega_{j} \subset I ; \omega_{j} \in \mathscr{C}_{k}} \int_{\omega_{j}} b_{l}(t) \phi_{k}^{\alpha_{l k}}(t) \\
& \times\left[\cot \left(\frac{\pi\left(x_{k}-t_{k}\right)}{p_{k}}\right)-\cot \left(\frac{\pi\left(x_{k}-t_{k}^{j}\right)}{p_{k}}\right)\right] d \mu(t),
\end{aligned}
$$

where $t^{j}=\left\{t_{k}^{j}\right\}_{k \geq 0}$ is any fixed point in $\omega_{j}$. (See the proof below (32) on p. 138 in [8].) By Minkowski's inequality for integrals, we have

$$
\begin{aligned}
{\left[\sum_{l}\left|H_{k}\left(b_{l} \phi_{k}^{\alpha_{l k}}\right)(x)\right|^{2}\right]^{1 / 2} \leq } & \frac{1}{2} \frac{1}{\mu(I)} \sum_{\omega_{j} \subset I ; \omega_{j} \in \mathscr{C}_{k}} \int_{\omega_{j}}\left[\sum_{l}\left|b_{l}(t)\right|^{2}\right]^{1 / 2} \\
& \times\left|\cot \left(\frac{\pi\left(x_{k}-t_{k}\right)}{p_{k}}\right)-\cot \left(\frac{\pi\left(x_{k}-t_{k}^{j}\right)}{p_{k}}\right)\right| d \mu(t) .
\end{aligned}
$$

Thus, for any coset $I$ of $G_{k}$, Fubini's theorem gives

$$
\begin{aligned}
\int_{I \cap c \Omega^{*}} & {\left[\sum_{l}\left|H_{k}\left(b_{l} \phi_{k}^{\alpha_{l k}}\right)(x)\right|^{2}\right]^{1 / 2} d \mu(x) } \\
\leq & \frac{1}{2} \frac{1}{\mu(I)} \sum_{\omega_{j} \subset I ; \omega_{j} \in \epsilon_{k}} \int_{\omega_{j}}\left[\sum_{l}\left|b_{l}(t)\right|^{2}\right]^{1 / 2} \\
& \times \int_{I \cap c \omega_{j}^{*}}\left|\cot \left(\frac{\pi\left(x_{k}-t_{k}\right)}{p_{k}}\right)-\cot \left(\frac{\pi\left(x_{k}-t_{k}^{j}\right)}{p_{k}}\right)\right| d \mu(x) d \mu(t) .
\end{aligned}
$$

Since, for $t \in \omega_{j}$, a simple computation gives

$$
\frac{1}{\mu(I)} \int_{I \cap c \omega_{j}^{*}}\left|\cot \left(\frac{\pi\left(x_{k}-t_{k}\right)}{p_{k}}\right)-\cot \left(\frac{\pi\left(x_{k}-t_{k}^{j}\right)}{p_{k}}\right)\right| d \mu(x) \leq C,
$$

we have

$$
\begin{aligned}
\int_{I \cap c \Omega^{*}}\left[\sum_{l}\left|H_{k}\left(b_{l} \phi_{k}^{\alpha_{l k}}\right)\right|^{2}\right]^{1 / 2} d \mu & \leq C \sum_{\omega_{j} \subset I ; \omega_{j} \in \mathbb{F}_{k}} \int_{\omega_{j}}\left(\sum_{l}\left|b_{l}\right|^{2}\right)^{1 / 2} d \mu \\
& \leq C \sum_{\omega_{j} \subset I ; \omega_{j} \in \mathbb{F}_{k}} \int_{\omega_{j}}\left(\sum_{l}\left|f_{l}\right|^{2}\right)^{1 / 2} d \mu
\end{aligned}
$$


by (2.7). Therefore

$$
\begin{aligned}
& \mu\{x\left.\notin \Omega^{*}: \sum_{k=0}^{\infty}\left[\sum_{l}\left|H_{k}\left(b_{l} \phi_{k}^{\alpha_{l k}}\right)(x)\right|^{2}\right]^{1 / 2}>y / 4\right\} \\
& \leq C y^{-1} \sum_{k=0}^{\infty} \int_{c \Omega^{*}}\left[\sum_{l}\left|H_{k}\left(b_{l} \phi_{k}^{\alpha_{l k}}\right)\right|^{2}\right]^{1 / 2} d \mu \\
& \leq C y^{-1} \sum_{k=0}^{\infty} \sum_{\omega_{j} \in \mathscr{C}_{k}} \int_{\omega_{j}}\left(\sum_{l}\left|f_{l}\right|^{2}\right)^{1 / 2} d \mu \\
& \leq C y^{-1}\left\|\left(\sum_{l}\left|f_{l}\right|^{2}\right)^{1 / 2}\right\|_{1} .
\end{aligned}
$$

This establishes (3.4), and hence completes the proof of Theorem 2.

\section{Proof of Theorem 1}

It suffices to prove (1.2). Let $f \in H^{1}(G)$ and $f_{k}=S_{m_{k+1}} f-S_{m_{k}} f, k=$ $0,1, \ldots$. For $m_{k} \leq n_{k}<m_{k+1}, S_{n_{k}} f=S_{m_{k}} f+S_{n_{k}} f_{k}$. Hence, for $y>0$,

$$
\mu\left\{\sup _{k \geq 0}\left|S_{n_{k}} f\right|>y\right\} \leq \mu\left\{\sup _{k \geq 0}\left|S_{m_{k}} f\right|>y / 2\right\}+\mu\left\{\sup _{k \geq 0}\left|S_{n_{k}} f_{k}\right|>y / 2\right\} .
$$

By (1.1),

$$
\mu\left\{\sup _{k \geq 0}\left|S_{m_{k}} f\right|>y / 2\right\} \leq 2 y^{-1}\left\|f^{*}\right\|_{1} \leq C y^{-1}\|f\|_{H^{1}} .
$$

From (1.3) of Theorem 2,

$$
\begin{aligned}
\mu\left\{\sup _{k \geq 0}\left|S_{n_{k}} f_{k}\right|>y / 2\right\} & \leq \mu\left\{\left(\sum_{k=0}^{\infty}\left|S_{n_{k}} f_{k}\right|^{2}\right)^{1 / 2}>y / 2\right\} \\
& \leq C y^{-1}\left\|\left(\sum_{k=0}^{\infty}\left|f_{k}\right|^{2}\right)^{1 / 2}\right\|_{1} \leq C y^{-1}\|f\|_{H^{1}} .
\end{aligned}
$$

This completes the proof of Theorem 1 .

\section{REFERENCES}

1. B. Davis, On the integrability of the martingale square function, Israel J. Math. 8 (1970), 187-190.

2. S. Fridli and P. Simon, On the Dirichlet kernels and a Hardy space with respect to the Vilenkin system, Acta Math. Hung. 45 (1985), 223-234.

3. A. M. Garsia, Martingale inequalities: Seminar notes on recent progress, Benjamin, Reading, Massachusetts, 1973.

4. J. A. Gosselin, Almost everywhere convergence of Vilenkin-Fourier series, Trans. Amer. Math. Soc. 185 (1973), 345-370. 
5. N. R. Ladhawala and D. C. Pankratz, Almost everywhere convergence of Walsh Fourier series of $\mathscr{H}^{1}$-functions, Studia Math. 59 (1976/77), 85-92.

6. P. Simon, Investigations with respect to the Vilenkin system, Ann. Univ. Sci. Budapest. Eötvös Sect. Math. 27 (1984), 87-101 (1985).

7. N. Ja. Vilenkin, On a class of complete orthonormal systems, Amer. Math. Soc. Transl. (2) 28 (1963), 1-35.

8. W.-S. Young, Mean convergence of generalized Walsh-Fourier series, Trans. Amer. Math. Soc. 218 (1976), 311-320.

Department of Mathematics, University of Alberta, Edmonton, Alberta T6G 2G1 CANADA 